Sven Haller

Niels Birbaumer

Ralf Veit

\title{
Real-time fMRI feedback training may improve chronic tinnitus
}

Received: 23 April 2009

Revised: 30 July 2009

Accepted: 5 August 2009

Published online: 16 September 2009

(C) European Society of Radiology 2009

\section{S. Haller $(\bowtie)$}

Institute of Radiology,

Department of Neuroradiology,

University Hospital Basel,

Petersgraben 4,

CH 4031 Basel, Switzerland

e-mail: shaller@uhbs.ch

Tel.: +41-61-2652525

Fax: +41-61-2654908

\section{S. Haller}

Institute of Neuroradiology,

Department of Imaging and Medical

Informatics, Geneva University

Hospital,

Geneva, Switzerland

\section{N. Birbaumer · R. Veit}

Institute of Medical Psychology and

Behavioral Neurobiology,

University of Tübingen,

Tübingen, Germany

\section{N. Birbaumer}

Ospedale San Camillo, Instituto di Ricovero e Cura a Carattere Scientifico, Venezia, Italy
Abstract Objectives: Tinnitus consists of a more or less constant aversive tone or noise and is associated with excess auditory activation. Transient distortion of this activation (repetitive transcranial magnetic stimulation, rTMS) may improve tinnitus. Recently proposed operant training in real-time functional magnetic resonance imaging (rtfMRI) neurofeedback allows voluntary modification of specific circumscribed neuronal activations. Combining these observations, we investigated whether patients suffering from tinnitus can (1) learn to voluntarily reduce activation of the auditory system by rtfMRI neurofeedback and whether (2) successful learning improves tinnitus symptoms. Methods: Six participants with chronic tinnitus were included. First, location of the individual auditory cortex was determined in a standard fMRI auditory block-design localizer. Then, participants were trained to voluntarily reduce the auditory activation (rtfMRI) with visual biofeedback of the current auditory activation. Results: Auditory activa- tion significantly decreased after rtfMRI neurofeedback. This reduced the subjective tinnitus in two of six participants. Conclusion: These preliminary results suggest that tinnitus patients learn to voluntarily reduce spatially specific auditory activations by rtfMRI neurofeedback and that this may reduce tinnitus symptoms. Optimized training protocols (frequency, duration, etc.) may further improve the results.

Keywords fMRI $\cdot$ BOLD · Biofeedback · Tinnitus ·

Neurofeedback
Abbreviations BOLD: blood oxygenation level dependent . DLPFC: dorso-lateral prefrontal cortex $\cdot$ EEG:
electroencephalography $\cdot$ EPI: echoplanar imaging · fMRI: functional magnetic resonance imaging $\cdot \mathrm{rtfMRI}$ : real-time functional magnetic resonance imaging - rTMS: repetitive transcranial magnetic stimulation - VLPFC: ventro-lateral prefrontal cortex

\section{Introduction}

Tinnitus consists of the auditory perception of sounds or noise not caused or triggered by external auditory stimuli, and affects millions of people [1]. It is estimated that in 1$3 \%$ of the general population tinnitus becomes chronic and sufficiently intrusive to interfere with patients' quality of life [2]. The underlying neuronal mechanism is only partially understood, and treatment options are limited [3]. It was shown that tinnitus is associated with over-activation within the auditory network [4-6]. Further, it was shown that repetitive transcranial magnetic stimulation (rTMS) over the auditory area that temporarily disrupts neuronal activations may alleviate tinnitus symptoms [6-8]. 
Biofeedback allows learning voluntary control over otherwise autonomous physiological parameters by means of operant training by providing real-time feedback of a particular physiological change. Biofeedback was first demonstrated for the autonomous nervous system (heart rate and skin conductance) in the 1950s [9]. In the nervous system, biofeedback (also called neurofeedback) classically uses electroencephalography (EEG), for example, to restore communication in severely paralyzed patients $[10$, 11]. Recently, real-time functional magnetic resonance imaging (rtfMRI) neurofeedback was introduced [12-15]. This non-invasive technique has a substantially higher spatial resolution compared to EEG [13]. We reason that this high spatial resolution of rtfMRI implies a substantial clinical power because it is possible to learn voluntary control over specific brain areas, while EEG feedback allows modifications of large cortical areas only. The majority of the few available rtfMRI biofeedback studies investigated the principal feasibility of rtfMRI neurofeedback and the neuronal mechanisms of neurofeedback in healthy volunteers $[13,14,16]$. To date, only one controlled rtfMRI group study in patients is available that demonstrates a beneficial effect of rtfMRI neurofeedback in chronic pain patients [15].

Combining the above-mentioned observations, we hypothesize that subjects with tinnitus can (1) learn to voluntarily reduce the hyperactivity of auditory brain areas by means of rtfMRI neurofeedback and that (2) this reduces tinnitus symptoms.

\section{Materials and methods}

\section{Subjects}

The study was approved by the local ethics committee of the Medical Faculty of the University of Tübingen, Germany. Six subjects (three females, three males, age $36.0 \pm 14.2$ years) gave their written informed consent prior to inclusion. All subjects had continuous, non-pulsating tinnitus (5 monaural, 1 binaural) for several years. Tinnitus self-assessments $[17,18]$ were performed before and after the MRI session.

\section{Task procedure}

First, a standard fMRI auditory block-design paradigm was performed to identify individual primary auditory cortices (ROI1) with $20 \mathrm{~s}$ ON, $20 \mathrm{~s}$ OFF bilateral auditory stimulation, with sine tone of $1,000 \mathrm{~Hz}$ pulsating at $6 \mathrm{~Hz}$. This stimulus is known to induce a strong and long-lasting BOLD response [19]. Thereafter, we applied four rtfMRI neurofeedback training sessions (each 4 min $24 \mathrm{~s}$ ). Each trial started with a 10-s baseline period followed by a block-design alternating between down-regulation and no- regulation periods lasting for $18 \mathrm{~s}$ each. To account for unspecific and global BOLD changes, we used a second ROI (ROI2) posterior and inferior to the primary auditory area at the temporo-occipital junction and provided the difference between the target auditory ROI1 and control ROI2 as feedback. We used two criteria for selecting the control region: (1) no activation during the auditory localizer session and (2) the area not involved in tinnitus or in the established rtfMRI experiments in healthy controls.

In the down-regulation trials, visual feedback was presented by means of thermometer bars [20]. During noregulation tasks, no feedback signal was given, and the thermometer display showed no activation changes. During the no-regulation condition, the subjects were asked to count back silently. The participants were informed about the data processing delay of $1.5 \mathrm{~s}$ and of the intrinsic physiological hemodynamic response delay of about $6 \mathrm{~s}$. The subjects were not instructed to use a certain strategy for down-regulation, and we recommended using a strategy that already helped them to reduce the intensity of the tinnitus noise in their daily life.

We used the Turbo BrainVoyager software package (www.brainvoyager.com) in combination with in-house Matlab (www.mathworks.com) scripts for real-time data analysis.

\section{fMRI data acquisition}

Functional images were acquired on a 3-T whole body MR scanner with a standard 12-channel head coil (Siemens Magnetom Trio Tim, Siemens Erlangen, Germany). A standard Echo-Planar Imaging sequence was used [EPI; TR (repetition time $)=1.5 \mathrm{~s}$, matrix size $=64 \times 64$, effective echo time TE (echo time) $=30 \mathrm{~ms}$, flip angle $\alpha=70^{\circ}$, bandwidth $=$ $1.954 \mathrm{kHz} /$ pixel; 16 slices; voxel size $=3.3 \times 3.3 \times 5.0 \mathrm{~mm}^{3}$, slice gap $=1 \mathrm{~mm}]$. Additionally, we acquired an anatomical T1-weighted whole brain scan (MPRAGE, matrix size $=$ $256 \times 256,160$ partitions, $1 \mathrm{~mm}^{3}$ isotropic voxels, TR= $2,300 \mathrm{~ms}, \mathrm{TE}=3.93 \mathrm{~ms}$, TI (inversion time) $=1,100 \mathrm{~ms}$, $\alpha=8^{\circ}$ ).

\section{Offline post-hoc fMRI data analysis}

The post-hoc, off-line data analysis was done with the SPM5 statistical parametric mapping software package (Wellcome Department of Imaging Neuroscience, London). Processing included spatial data smoothing (8-mm Gaussian kernel), temporal drift removal $(0.0088-\mathrm{Hz}$ high-pass filtering) and spatial normalization to the Montreal Neurological Institute (MNI) space. The six movement regressors were used as confounds to reduce movement-related variance. All conditions were modeled with a canonical hemodynamic response function (HRF) using standard SPM 
5 settings. The following contrasts were analyzed: downregulation vs. no-regulation and no-regulation vs. downregulation. We performed two major analyses.

At the single subject level, we tested the within-subject learning effects by estimating a linear decrease in activity over sessions (Table 1). The reported single-subject p-values were not corrected for multiple comparisons, because we describe single-subject effects. All identified significant voxels were within the ROIs of the auditory localizer runs.

At the group level, a fixed-effects group analysis was performed using the last session of each subject testing for the amount of successful down-regulation at the end of the training, i.e., contrasting the down-regulation trials versus the no-regulation tasks (Fig. 2a,b,e,f,g, Table 2). Effects were considered as significant using a whole-brain familiywise error rate (FWE) of $p<0.001$. Additionally, we performed a linear regression t-test analysis of the individual BOLD responses in the auditory areas separately for the right and left auditory areas with session number as the independent and the parameter estimates of each subject as the dependent variable (Fig. 2c,d). This test was performed one-tailed under the strict a priori hypothesis of decreasing activations over training sessions.

\section{Results}

Single subject analysis

Five of the six included subjects successfully learned to down-regulate their activations in the auditory ROI. An example subject is illustrated in Fig. 1. The individual peak areas with linear signal decrease over time are listed in Table 1.

Table 1 depicts the test of linear signal decrease over training sessions at the individual level: peak activation of the activation cluster in MNI standard space, corresponding t-value and p-value

\section{Group analysis}

Regression analyses revealed a significant linear decrease in the auditory activations over the training sessions $(p<0.05$ bilaterally; Fig. 2 c, d). The group analysis of the last training session shows a significant decrease in activation after rtfMRI neurofeedback training in bilateral auditory areas (Fig. 2 a, b; Table 2). Moreover we found deactivations in the occipital lobe and in areas comprising the default brain network during resting states (prefrontal cortex, precuneus, inferior parietal lobe). Note that this decrease in activation is spatially restricted to the abovementioned areas, not a general decrease in neuronal activations of the whole brain. Increased activations during rtfMRI neurofeedback training were found in bilateral insula extending to the ventro-lateral prefrontal cortex [VLPFC, the right dorso-lateral prefrontal cortex (DLPFC) anatomical labeled as middle frontal gyrus and right occipito-temporal junction].

\section{Behavioral data}

Subjects reported no change $(\mathrm{N}=4)$ or mild improvement $(\mathrm{N}=2)$ in tinnitus symptoms after rtfMRI neurofeedback (follow-up questionnaire approximately 2 weeks after training). None of the patients reported increasing tinnitus symptoms.

\section{Discussion}

The present investigation is based on the combination of three previous findings: (1) tinnitus is associated with

(uncorrected). Except for subject 3, all subjects successfully decreased the activations by means of rtfMRI neurofeedback training

\begin{tabular}{|c|c|c|c|c|c|c|}
\hline \multirow[t]{2}{*}{ Subject } & \multirow[t]{2}{*}{ Side } & \multicolumn{3}{|c|}{ MNI coordinates } & \multirow[t]{2}{*}{ t-value } & \multirow[t]{2}{*}{ p-value } \\
\hline & & $\mathrm{x}$ & $\mathrm{y}$ & $\mathrm{z}$ & & \\
\hline \multirow[t]{2}{*}{1} & Left & -39 & -33 & 15 & $\mathrm{t}=7.05$ & $\mathrm{p}<0.0001$ \\
\hline & Right & 48 & -24 & 9 & $t=6.65$ & $\mathrm{p}<0.0001$ \\
\hline \multirow[t]{2}{*}{2} & Left & -48 & -30 & 9 & $t=7.68$ & $\mathrm{p}<0.0001$ \\
\hline & Right & 60 & -21 & 12 & $\mathrm{t}=8.09$ & $\mathrm{p}<0.0001$ \\
\hline \multirow[t]{2}{*}{3} & Left & Not & & & & \\
\hline & Right & Not & & & & \\
\hline \multirow[t]{2}{*}{4} & Left & -39 & -33 & 18 & $t=2.61$ & $\mathrm{p}=0.005$ \\
\hline & Right & 48 & -30 & 9 & $\mathrm{t}=3.13$ & $\mathrm{p}=0.001$ \\
\hline \multirow[t]{2}{*}{5} & Left & -36 & -27 & 27 & $\mathrm{t}=3.30$ & $\mathrm{p}=0.001$ \\
\hline & Right & Not & & & & \\
\hline \multirow[t]{2}{*}{6} & Left & -45 & -21 & 21 & $\mathrm{t}=3.12$ & $\mathrm{p}=0.001$ \\
\hline & Right & 42 & -27 & 21 & $\mathrm{t}=2.92$ & $\mathrm{p}=0.002$ \\
\hline
\end{tabular}


Table 2 Lists those areas with a significant decrease in activation (top) or increase (bottom) as a result of the rtfMRI neurofeedback training sessions. Brain region of the decreasing/increasing activa- tion, peak coordinate $\mathrm{x}, \mathrm{y}, \mathrm{z}$ in Montreal Neurological Institute (MNI) space and t-value (family-wise error, FWE, rate corrected, $\mathrm{p}<0.001$ )

\begin{tabular}{|c|c|c|c|c|}
\hline \multirow[t]{2}{*}{ Brain regions } & \multicolumn{3}{|c|}{ MNI coordinates } & \multirow[t]{2}{*}{ t-value } \\
\hline & $\mathrm{x}$ & $\mathrm{y}$ & $\mathrm{z}$ & \\
\hline \multicolumn{5}{|c|}{ Decreasing activation during rtfMRI training } \\
\hline Occipital lobe $\mathrm{R}$ & 27 & -96 & 6 & 8.99 \\
\hline Occipitallobe L & -30 & -93 & -3 & 8.24 \\
\hline Precuneus & 0 & -54 & 42 & 8.74 \\
\hline Angular gyrus $\mathrm{R}$ & 45 & -69 & 39 & 8.21 \\
\hline Angular gyrus L & -42 & -69 & 39 & 6.80 \\
\hline Superior medial frontal cortex $\mathrm{L}$ & -9 & 60 & 33 & 7.13 \\
\hline Superior medial frontal cortex $\mathrm{R}$ & 0 & 66 & 24 & 6.65 \\
\hline Heschl gyrus R & 39 & -27 & 18 & 6.15 \\
\hline Heschl gyrus R & 60 & -12 & 15 & 5.51 \\
\hline Heschl gyrus L & -57 & -12 & 18 & 5.05 \\
\hline Middle temporal gyrus L & -60 & -48 & -9 & 5.24 \\
\hline \multicolumn{5}{|c|}{ Increasing activation during rtfMRI training } \\
\hline Insula $\mathrm{R}$ & 33 & 27 & 0 & 7.96 \\
\hline Middle temporal gyrus $\mathrm{R}$ & 39 & -66 & 15 & 7.18 \\
\hline Frontal Inferior opercularis $\mathrm{R}$ & 51 & 9 & 21 & 6.98 \\
\hline Middle frontal gyrus $\mathrm{R}$ & 36 & 42 & 21 & 5.69 \\
\hline Insula L & -36 & 18 & -6 & 6.26 \\
\hline Middle occipital L & -30 & -75 & 21 & 5.55 \\
\hline
\end{tabular}

excess auditory activation [4-6]; (2) transient reduction or interruption of this activation (repetitive transcranial magnetic stimulation, TMS) may improve tinnitus [6-8]; (3) realtime fMRI neurofeedback allows learning the voluntary control of localized neuronal activations through operant training [12-15].

In accordance with our hypothesis, patients with tinnitus successfully learned to reduce the auditory activations by means of rtfMRI neurofeedback. This improved the subjective tinnitus symptoms in some patients. The current proof of principle results justify future rtfMRI neurofeedback studies in tinnitus in larger cohorts and adequate controls.

Concerning the brain activations, rtfMRI neurofeedback training induced a significant decrease in the activations of the auditory cortex despite the small sample size of only six participants, which implies a high potential effect of rtfMRI neurofeedback. Our data support findings of recent studies showing that with rtfMRI-neurofeedback people can learn to control changes of their BOLD signal in circumscribed brain areas within a few sessions $[14,15,20]$. Only one of six participants did not manage to down-regulate the activations in the primary auditory cortex.

Although the task of the present study was to decrease activations within the auditory cortex as discussed above, the post-hoc data analysis identified additional and spatially separated regions with decreasing activations, although these areas were not included in the feedback display of the rtfMRI training: the medial frontal cortex, the precuneus and the angular gyrus extending to the inferior parietal lobe. These areas are part of the 'default brain network' [21, 22] and consistently active during resting states or show deactivations during cognitively demanding tasks [22, 23]. Conversely, increasing activations were found in the insula extending to the bilateral VLPFC and the right DLPFC. The increasing activation within these regions during down-regulation of the primary auditory area might suggest a functional role of these areas in the down-regulation process. These areas were reported during self-regulation of emotional responses using cognitive reappraisal strategies or during diverting attention from emotional arousal stimuli $[24,25]$. It was proposed that the insula might play a critical role in mediating the influence of peripheral autonomic arousal on consciously experienced emotional states [26, 27]. Correspondingly, the subjects in the present study primarily used positive events (remembering pleasurable events like a holiday) or relaxation-related strategies ('autogenic training') to decrease the activation in the auditory cortex.

Concerning the behavioral effects, we cannot expect that in this proof-of-concept study four training sessions within a single day will evoke a strong effect, in particular because tinnitus is a chronic condition lasting for several years in 
Fig. 1 Illustrates the experimental setup for a single subject. First, a standard fMRI blockdesign auditory localizer in combination with real-time data analysis was used to identify the individual auditory areas (a, screen-shot of the real-time data analysis using Turbo Brainvoyager, www.brainvoyager. com). The right auditory area (green square) and left auditory area (red square) illustrate a clear task-related BOLD response (graphs on the right-hand side with baseline periods in blue and auditory stimulation periods in green). Note the hemodynamic delay of the BOLD response. The third, lowest graph on the right-hand side illustrates the on-line motion correction. In a second step, the individually defined auditory areas were used for the rtfMRI neurofeedback. A thermometer bar was visually presented to the subjects inside the MRI scanner (b) that indicated the current BOLD activation in the auditory region of interest. Subjects trained to down-regulate this activation. The presented example illustrates a current down-regulation (thermometer below the mean). The post-hoc data analysis of a single subject depicts those areas that are down-regulated after the rtfMRI biofeedback training (c). Additionally, the evolution of the BOLD activations, illustrated as BETA estimate contrast (in arbitrary units) over the four training sessions, is illustrated for left (d, at locations $\mathrm{X}-39, \mathrm{Y}-33$, Z 12 in Montreal Neurological Institute MNI space) and right (e, at locations X 48, Y -24, Z 9) auditory areas
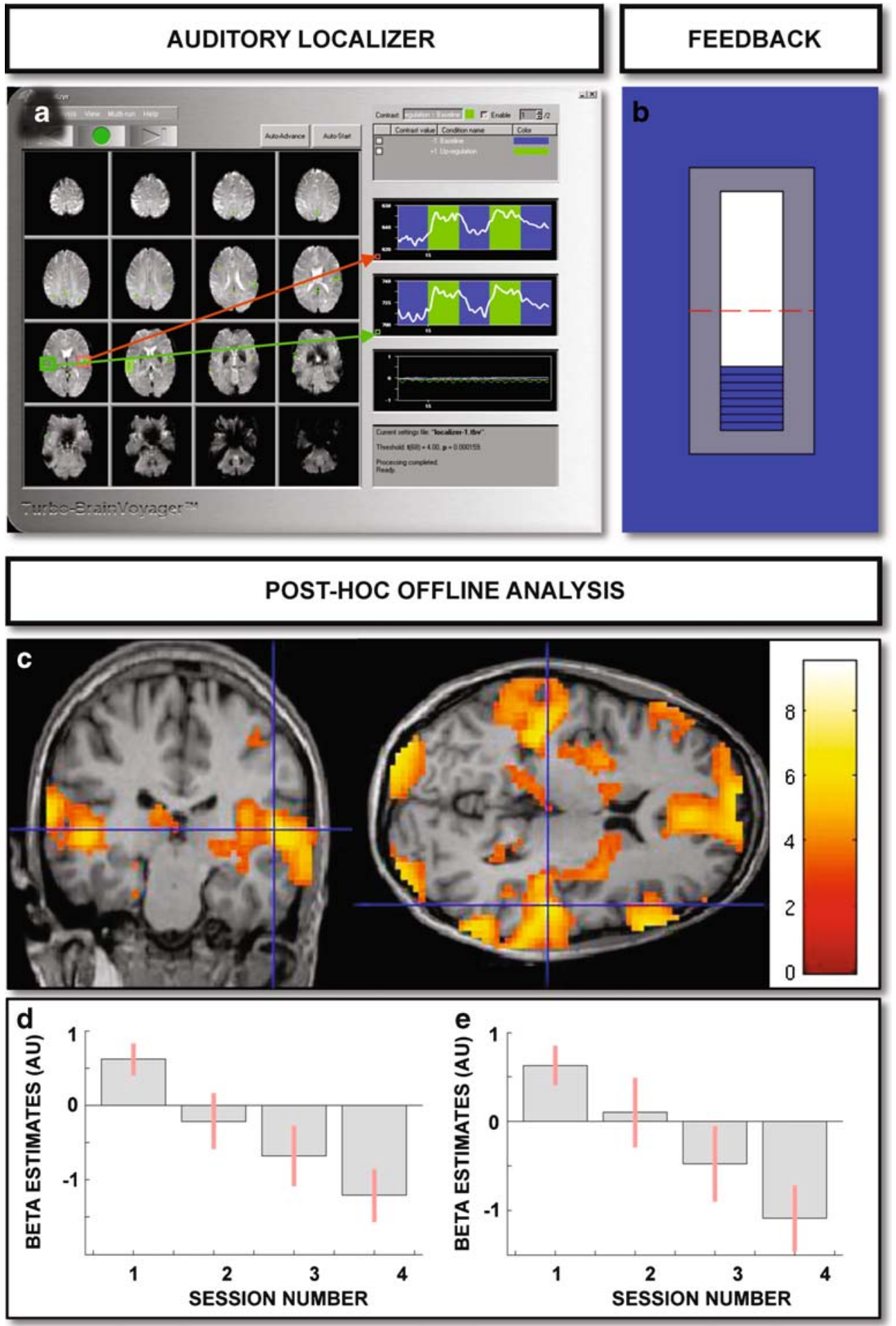

our patients. Correspondingly, the mean activation continuously decreased within the first four training sessions (Fig. 2 c,d). On the other hand, the observed positive minor behavioral effect despite only 1 training day suggests a substantial potential effect of rtfMRI neurofeedback in tinnitus. Necessary optimizations of
rtfMRI training include adjustment of the number, duration and frequency of the rtfMRI training sessions. Learning is difficult in the absence of guidelines for mental strategies and can lead to a drop of motivation, especially in the uncomfortable environment of the MRI scanner [15, 28]. The rtfMRI biofeedback algorithm has 
Fig. 2 Illustrates the effect of the $\mathrm{rtfMRI}$ neurofeedback training of the group analysis $(\mathrm{N}=6)$. Decreasing activations after rtfMRI neurofeedback are present in the bilateral auditory area (yellow circles in $\mathbf{a}$ and $\mathbf{b}$ ) and in the default brain network (prefrontal cortex, precuneus, inferior parietal lobe). The additional linear regression analysis of the activations in the left (c) and right (d) auditory area demonstrates a significant decrease over the four training sessions. Increasing activations (E-F) were present in bilateral insula extending to the bilateral VLPFC and right DLPFC, and right occipito-temporal junction. SPM convention, left hemisphere on left hand side
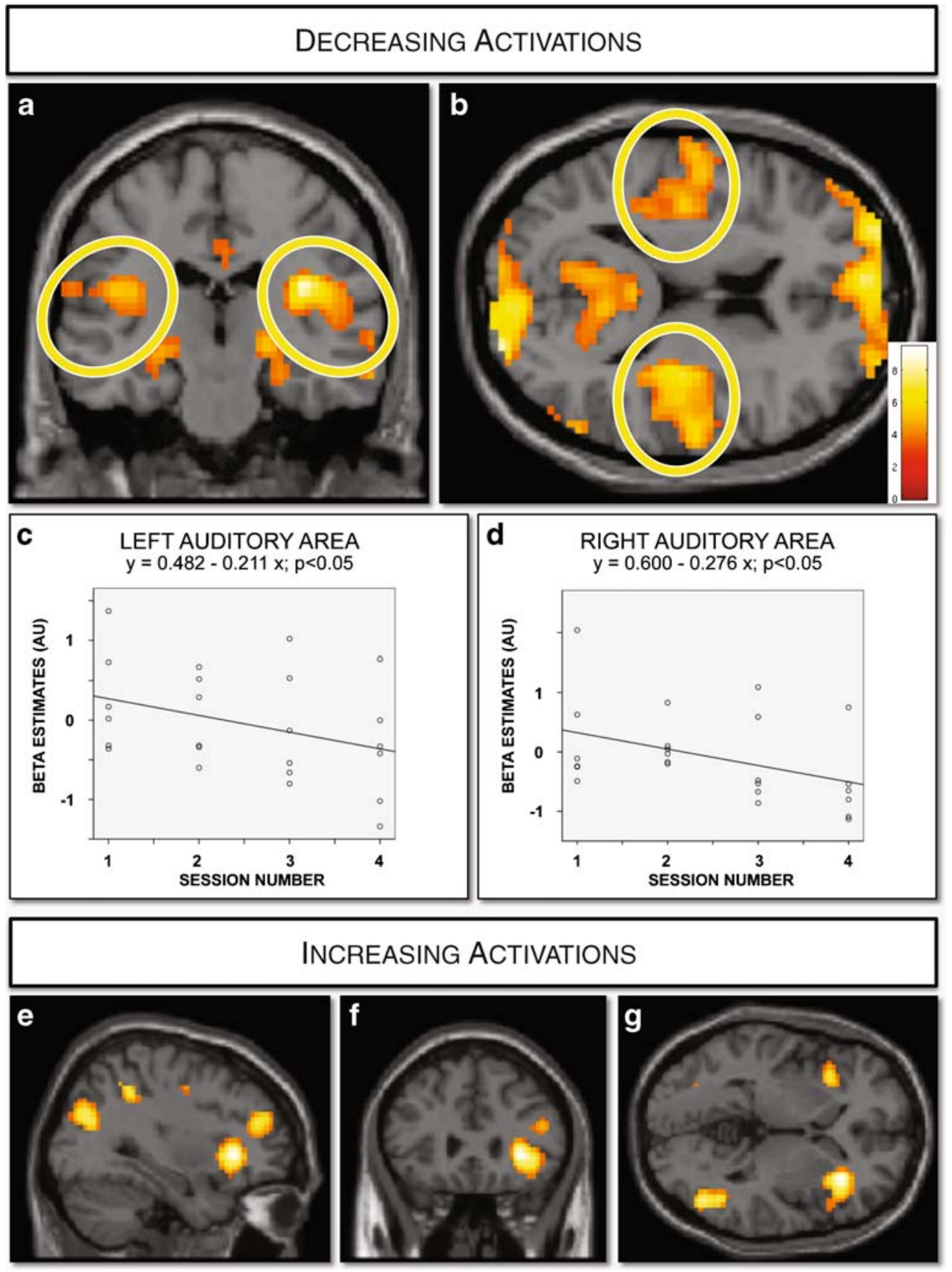

to be optimized, and the training protocol should be adapted to the individual patient.

Previous rTMS studies improved tinnitus [6-8]; however, the effect is transient and depends on the presence of the rTMS device. The rtfMRI neurofeedback technique has the advantage that once a participant has learned to control the individual auditory activations, this strategy can be used in every day life to improve tinnitus. Previous EEG biofeedback studies in tinnitus successfully improved tinnitus symptoms by up-regulating alpha-activity and down-regulating beta-activity [29] or by enhancement of tau activity within the alpha frequency range and concomitant reduction in delta power [30]. As compared to these EEG studies, the major advantage of rtfMRI biofeedback is the much higher spatial specificity that allows for selective reduction of the auditory activation only.

This proof of principle study aims to illustrate the clinical potential of rtfMRI neurofeedback. Tinnitus was chosen as an example because of the existing clear apriori hypotheses and the localized target region. The presented principle of rtfMRI neurofeedback represents a novel 'therapeutic' instead of the usual diagnostic application of 
MRI. This principle might be transferred to other diseases with presumed excess neuronal activations, such as auditory or visual hallucinations. Likewise, rtfMRI biofeedback might also be used to increase neuronal activations, e.g., in stroke patients with motor disorders, as increased motor activity might improve motor dysfunctions [31].

A major limitation of the presented proof of concept investigation evidently is the small sample size.

The frequency of the auditory localizer stimulus was identical in all subjects despite differences in the subjective tinnitus frequency. Given the established tonotopic organization of the auditory cortex, [32], we reason that the auditory stimulus of the functional localizer experiment should ideally match the subjective tinnitus to detect the exact sub-region of the auditory area. Due to the comparably small distance of the tonotopic distribution in relation to size of the volumes-of-interest, future studies are needed to determine whether the additional effort of generating individually tuned auditory stimuli outweigh the more convenient use of a standardized "fixed" auditory stimuli, in particular with respect to potential clinical applications of the presented technique.

A general concern in rtfMRI neurofeedback is related to the spatial specificity of the learned modification of brain activations. The BOLD response is influenced, for example, by respiration and the related blood level of carbon dioxide [33]. Subjects might simply hyperventilate to reduce the BOLD activations that also-but not specifically - includes the auditory regions. To control for global effects, we provided feedback as the difference between the target auditory area against a distant control region. Additionally, the post-hoc group comparison of brain activations after rtfMRI training versus prior to training (Fig. 2) confirms that the subjects learned a regionspecific down-regulation of the auditory areas.

Another limitation is the absence of control groups. The only available clinical rtfMRI group study found a beneficial effect of neurofeedback in patients $(\mathrm{N}=8)$ with chronic pain [15]. The authors applied pain (noxious thermal stimulus) in healthy controls using 'true' rtfMRI neurofeedback as well as (1) training, without rtfMRI information, (2) purely behavioral training, (3) rtfMRI information derived from a brain region not involved in affective pain processing and (4) rtfMRI information of other subjects (false or sham feedback). None of these healthy control groups without the true rtfMRI neurofeedback was able to reduce the perceived pain in response to the noxious thermal stimulus.

\section{Conclusions}

In conclusion, we could prove the principle that patients with tinnitus successfully learned to reduce the auditory activations by real-time functional magnetic resonance imaging neurofeedback and that this improved tinnitus symptoms in at least two out of six patients.

Acknowledgements We thank all subjects for participation in the study.

Conflict of interest No conflicts of interest.

\section{References}

1. Heller AJ (2003) Classification and epidemiology of tinnitus. Otolaryngol Clin North Am 2:239-248

2. Dobie RA (2003) Depression and tinnitus. Otolaryngol Clin North Am 2:383-388

3. Dobie RA (1999) A review of randomized clinical trials in tinnitus. Laryngoscope 8:1202-1211

4. Muhlnickel W, Elbert T, Taub E et al (1998) Reorganization of auditory cortex in tinnitus. Proc Natl Acad Sci U S A 17:10340-10343

5. Andersson G, Lyttkens L, Hirvela $C$ et al (2000) Regional cerebral blood flow during tinnitus: a PET case study with lidocaine and auditory stimulation. Acta Otolaryngol 8:967-972
6. Kleinjung T, Eichhammer P, Langguth B et al (2005) Long-term effects of repetitive transcranial magnetic stimulation (rTMS) in patients with chronic tinnitus. Otolaryngol Head Neck Surg 4:566-569

7. Rossi S, De Capua A, Ulivelli M et al (2007) Effects of repetitive transcranial magnetic stimulation on chronic tinnitus. A randomised, cross over, double blind, placebo-controlled study. J Neurol, Neurosurg Psychiatry

8. Plewnia C, Reimold M, Najib A et al (2007) Dose-dependent attenuation of auditory phantom perception (tinnitus) by PET-guided repetitive transcranial magnetic stimulation. Hum Brain Mapp $3: 238-246$

9. Miller NE (1975) Clinical applications of biofeedback: Voluntary control of heart rate, rhythm, and blood pressure. In: Russel HI (ed) New horizons in cardiovascular practice. University Park Press, Baltimore, pp 239-249
10. Kubler A, Kotchoubey B, Hinterberger $\mathrm{T}$ et al (1999) The thought translation device: a neurophysiological approach to communication in total motor paralysis. Exp Brain Res 2:223-232

11. Birbaumer N, Ghanayim N, Hinterberger T et al (1999) A spelling device for the paralysed. Nature 6725:297-298

12. Weiskopf N, Veit R, Erb M et al (2003) Physiological self-regulation of regional brain activity using real-time functional magnetic resonance imaging (fMRI): methodology and exemplary data. Neuroimage 3:577-586

13. Weiskopf N, Scharnowski F, Veit R et al (2004) Self-regulation of local brain activity using real-time functional magnetic resonance imaging (fMRI). J Physiol Paris 4-6:357-373 
14. Weiskopf N, Sitaram R, Josephs O et al (2007) Real-time functional magnetic resonance imaging: methods and applications. Magn Reson Imaging 6:989-1003

15. deCharms RC, Maeda F, Glover GH et al (2005) Control over brain activation and pain learned by using real-time functional MRI. Proc Natl Acad Sci U S A 51:18626-18631

16. deCharms RC (2007) Reading and controlling human brain activation using real-time functional magnetic resonance imaging. Trends $\mathrm{Cogn} \mathrm{Sci}$ $11: 473-481$

17. Zenner HP, De Maddalena H (2005) Validity and reliability study of three tinnitus self-assessment scales: loudness, annoyance and change. Acta oto-laryngologica 11:1184-1188

18. Goebel G, Hiller W (1994) [The tinnitus questionnaire. A standard instrument for grading the degree of tinnitus. Results of a multicenter study with the tinnitus questionnaire]. $\mathrm{HNO}$ $3: 166-172$
19. Seifritz E, Esposito F, Hennel F et al (2002) Spatiotemporal pattern of neural processing in the human auditory cortex. Science 5587:1706-1708

20. Caria A, Veit R, Sitaram R et al (2007) Regulation of anterior insular cortex activity using real-time fMRI. Neuroimage 3:1238-1246

21. Biswal B, Yetkin FZ, Haughton VM et al (1995) Functional connectivity in the motor cortex of resting human brain using echo-planar MRI. Magn Reson Med 4:537-541

22. Damoiseaux JS, Rombouts SA, Barkhof $F$ et al (2006) Consistent resting-state networks across healthy subjects. Proc Natl Acad Sci U S A 37:13848-13853

23. Raichle ME, MacLeod AM, Snyder AZ et al (2001) A default mode of brain function. Proc Natl Acad Sci U S A 2:676-682

24. Ochsner KN, Ray RD, Cooper JC et al (2004) For better or for worse: neural systems supporting the cognitive downand up-regulation of negative emotion. Neuroimage 2:483-499

25. Eippert F, Veit R, Weiskopf $\mathrm{N}$ et al (2007) Regulation of emotional responses elicited by threat-related stimuli. Hum Brain Mapp 5:409-423

26. Critchley HD, Wiens S, Rotshtein P et al (2004) Neural systems supporting interoceptive awareness. Nat Neurosci 2:189-195
27. Critchley HD, Melmed RN, Featherstone E et al (2002) Volitional control of autonomic arousal: a functional magnetic resonance study. Neuroimage 4:909-919

28. Sitaram R, Caria A, Veit R et al (2007) FMRI brain-computer interface: a tool for neuroscientific research and treatment. Comput Intell Neurosci 25487

29. Gosepath K, Nafe B, Ziegler E et al (2001) [Neurofeedback in therapy of tinnitus]. HNO 1:29-35

30. Dohrmann K, Weisz N, Schlee W et al (2007) Neurofeedback for treating tinnitus. Prog Brain Res 473-485

31. Nelson LA (2007) The role of biofeedback in stroke rehabilitation: past and future directions. Top Stroke Rehabil 4:59-66

32. Bilecen D, Scheffler K, Schmid N et al (1998) Tonotopic organization of the human auditory cortex as detected by BOLD-FMRI. Hear Res 1-2:19-27

33. Cohen ER, Ugurbil K, Kim SG (2002) Effect of basal conditions on the magnitude and dynamics of the blood oxygenation level-dependent fMRI response. J Cereb Blood Flow Metab 9:1042-1053 